\title{
NOVAS DIMENSÕES DA POLÍTICA: PROTOCOLOS E CÓDIGOS NA ESFERA PÚBLICA INTERCONECTADA
}

\author{
Sergio Amadeu da Silveira
}

\begin{abstract}
RESUMO
O texto propõe a existência de uma divisão básica na relação entre política e Internet: a política "da" Internet e a política "na" Internet. Em seguida, agrega os temas das políticas da internet em três campos de disputas fundamentais: sobre a infra-estrutura da rede; sobre os formatos, padrões e aplicações; e sobre os conteúdos. Analisa os temas politicos atuais mais relevantes de cada campo conflituoso, articulando dois aspectos: o tecno-social e o jurídico-legislativo. Este artigo trabalha com o conceito de arquitetura de poder, uma extensão da definição de Alexander Galloway sobre o gerenciamento protocolar, e com a perspectiva de Yochai Benkler sobre a existência de uma esfera pública interconectada. Sua conclusão indica que a liberdade nas redes cibernéticas depende da existência da navegação não-identificada, ou seja, do anonimato. Apontada como uma das principais contendas políticas da Internet, a possibilidade do anonimato é apresentada como um elemento fundamental de resistência frente às amplas possibilidades de controle da comunicação social. Sendo assim, a garantia da liberdade de produzir conteúdos e de acessálos nas redes cibernéticas - em que os rastros digitais acompanham toda a navegação dos cidadãos interagentes - exige a defesa da não-identificação.
\end{abstract}

PALAVRAS-CHAVE: política da Internet; arquiteturas de poder; ciberpolítica; poder nas redes.

\section{INTRODUÇÃO}

Há diversas maneiras de discutir a relação entre a política e a Internet, mas existe uma divisão básica que amplia nossa clareza sobre o contexto histórico em que tais articulações ocorrem. Alguns observam a política $n a$ Internet, outros observam a política $d a$ Internet. Este artigo tratará das disputas sobre a organização e a ação das redes digitais e do conflito entre tentativas de controle e de emancipação do ciberespaço, bem como, da relevância dos códigos e protocolos para a comunicação livre e distribuída ou para a subordinação dos fluxos informacionais às tradicionais hierarquias e verticalidades que conformaram o mundo da comunicação de massas. Assim, aqui será discutida a política $d a$ Internet.

Sem dúvida, são cada vez mais relevantes para uma sociedade em rede (CASTELLS, 1998) as investigações e análises da política na Internet, principalmente agora, quando não há mais dúvidas de sua importância como arranjo midiático fundamental para as disputas pelo poder de Estado. A campanha de Barack Hussein Obama não se tratou de um ponto fora da curva, mas de uma tendência. Segundo a pesquisa realizada pelo $P e w$ Research Center for the People \& the Press e da Pew Internet \& American Life Project, em 2008, $42 \%$ dos jovens norte-americanos entre 18 e 29 anos disseram informar-se sobre a campanha pela Internet, destacando o papel crescente das chamadas redes sociais, principalmente, do MySpace e do Facebook (KHOUT, 2008).

Os estudos da política na rede focalizam, principalmente, o uso da Internet pelos partidos e candidatos (BARROS FILHO, COUTINHO \& SAFLATE, 2007; CHAIA, 2008). Existem, ainda, instigantes análises sobre o impacto das interfaces colaborativas (RHEINGOLD, 2004; UGARTE, 2008), do uso das mídias sociais como organizadores coletivos (SILVEIRA, 2007), do surgimento de uma nova esfera pública agora interconectada (BENKLER, 2006), além de uma vasta gama de debates sobre a representação, a organização política e as instituições democráticas a partir do ciberespaço (COLEMAN, 1999; HAGUE \& LOADER, 1999; NIXON \& JOHANSSON, 1999; WILHELM, 1999; BARBER, 2004; TERRANOVA, 2004; JENKINS \& THORBURN, 2004; NOVECK, 2005). 
Quando a opção é o estudo da política da rede, o temário e os objetos de análise passam a ser outros. Uma tentativa razoável de compreendêlos se dá a partir do seu agrupamento em três tipos de política: 1) sobre a infra-estrutura; 2) sobre os conteúdos; e 3) sobre os formatos e aplicações. Existem conflitos, disputas e proposições envolvendo agentes sociais em cada um desses agrupamentos. Diversos interesses chocam-se com a finalidade de manter ou alterar a dinâmica e a organização da Internet. Como a rede mundial de computadores torna-se extremamente relevante para o cotidiano comunicacional do planeta, identificar essa nova dimensão da política é igualmente relevante para compreendermos nosso tempo.

\section{CARACTERIZAÇÃO DA COMUNICAÇÃO EM REDES DIGITAIS}

A Internet é um arranjo midiático. Foi e é desenvolvida a partir da reunião de diversos meios e tecnologias. Manuel Castells descreveu a Internet como a "improvável intersecção da big science, da pesquisa militar e da cultura libertária" (CASTELLS, 2003, p. 19). Isso quer dizer que a rede, originada nos interesses militares, foi sendo reconfigurada pelos seus usuários, principalmente pela comunidade acadêmica e pelos hackers, grupos de programadores talentosos altamente influenciados pela contracultura da década de 1960. A rede mundial de computadores não pode ser definida como um produto nem como um conjunto de equipamentos com a evolução controlada por uma ou algumas empresas. A Internet não tem um centro, uma sede, trata-se de uma rede distribuída, construída colaborativamente por grupos de voluntários que no decorrer de sua história foram envolvendo, além dos acadêmicos e hackers, engenheiros e especialistas de várias empresas, para construírem os elementos fundamentais do funcionamento da rede, a saber, os seus protocolos de comunicação.

A evolução da Internet não foi ditada nem mesmo pelo governo norte-americano. A rede cresceu e foi moldada incorporando as soluções tecnológicas desenvolvidas pelos seus usuários. Como a Internet não estava submetida às hierarquias tradicionais das firmas (BENKLER, 2006), não era e não é necessário obter autorização de ninguém para criar nela novos conteúdos, formatos e tecnologias. Esse modelo aberto e não-proprietário é um dos fatores vitais que asseguraram a rápida expansão e evolução da rede com a con- tínua incorporação de novas criações tecnológicas. Até este momento, a Internet tem sido uma obra inacabada, em constante desenvolvimento. Tal situação desagrada vários segmentos econômicos e políticos do capitalismo, bem como extratos burocráticos de formação autoritária. A velha indústria cultural e o conjunto das indústrias de intermediação são diretamente afetadas, embora em diferentes graus, pela expansão da comunicação distribuída e pela surpreendente criatividade tecnológica espalhada pelo planeta. Entre inúmeros exemplos, é perceptível que o instituto da propriedade intelectual, muito importante para o capitalismo industrial, foi abalado por uma série de inovações realizadas pelos usuários da rede. $\mathrm{O}$ exemplo típico é o das redes $P 2 P$ (peer-to-peer), que ganhou destaque com o Napster no início deste século.

Em um processo de comunicação sob controle completo de Estados ou de firmas seria muito mais fácil conter tecnologias que colocassem em risco negócios bilionários ou interesses poderosos. Até o advento da comunicação distribuída em redes digitais, o capital teve maior poder sobre a comunicação. No livro Mudança estrutural da esfera pública (1984), Habermas mostrou como a imprensa foi capturada pelo capital, uma vez que as verbas de publicidade eram essenciais à manutenção das empresas de comunicação. De modo distinto, na comunicação em rede, nem mesmo as poderosas operadoras de telefonia puderam evitar que a disseminação da "voz sobre IP" (VoIP) retirasse seus rendimentos. A Recording Industry Association of America (RIAA), se conseguiu judicialmente impedir o funcionamento do Napster, fracassou ao tentar bloquear o uso das redes $P 2 P$ de compartilhamento de arquivos.

Nesse sentido, o professor Alejandro Piscitelli desenvolveu uma caracterização geral extremamente clara do que a rede mundial de computadores representa: "Internet fue el primer medio massivo de la historia que permitió una horizontalización de las comunicaciones, una simetría casi perfecta entre producción y recepción, alterando en forma indeleble la ecología de los medios"1 (PISCITELLI, 2002, p. 207). Já o ciberativista espanhol David

\footnotetext{
1 Tradução livre: "A Internet foi o primeiro meio massivo da história que permitiu a horizontalização das comunicações, uma simetria quase perfeita entre produção e recepção, alterando de forma indelével a ecologia dos meios".
} 
Ugarte difere a comunicação descentralizada da comunicação distribuída. Para ele, a Internet é uma rede distribuída. Ugarte utiliza a definição de Alexander Bard e Jan Söderqvist, que afirmam existir uma rede distribuída quando "todo ator individual decide sobre si mesmo, mas carece de capacidade e da oportunidade para decidir sobre qualquer dos demais atores" (Bard e Söderqvist apud UGARTE, 2008, p. 35).

Assim, é possível destacar as seguintes características da Internet em relação aos tradicionais meios de comunicação de massas:

\section{QUADRO 1 - COMPARAÇÃO ENTRE A INTERNET E OS MEIOS TRADICIONAIS DE COMUNICAÇÃO}

\begin{tabular}{|c|c|}
\hline RÁDIO-JORNAIS-TV & INTERNET \\
\hline Unidirecional & Multidirecional \\
Baixa interatividade & Alta interatividade \\
Hierárquica & Enredada \\
Verticalizada & Horizontalizada \\
Centralizada & Distribuída \\
Linear & Hipertextual \\
Analógica & Digital \\
Nacional/local & Transnacional/local \\
\hline
\end{tabular}

FONTE: O autor, a partir de Piscitelli (2002) e Ugarte (2008).

A multidirecionalidade da comunicação na Internet articulada à digitalização de toda produção simbólica e icônica da sociedade é uma das principais bases técnicas da alta interatividade. A horizontalização da comunicação enredada é garantida pela arquitetura da rede distribuída. Para compreender a comunicação em rede é preciso observar e acompanhar a sua arquitetura. Esta pode ser definida como o conjunto dos protocolos e a topologia de uma rede. Protocolos são regras que definem como os dados serão organizados, transferidos, armazenados, enfim, definem todas as regras de comunicação entre os elementos que participam da rede. A topologia é o desenho da rede, ou seja, como os pontos e nós estão estruturados. Na comunicação em rede, as possibilidades, os limites e o controle estão nas suas arquiteturas, códigos e protocolos. O jurista Lawrence Lessig alertou que, no ciberespaço, o código é a lei (LESSIG, 1999).
O controle da comunicação através de uma organização de hierarquia verticalizada não ocorre na Internet por se tratar de uma rede distribuída, conforme o argumento de David Ugarte (UGARTE, 2008, p. 35). Todavia, há muito tempo, alguns alertaram para a existência de hierarquias de conexões que concentrariam os fluxos da rede (RIBEIRO, 2000). Focalizando os protocolos, a abordagem de Galloway permite esclarecer bem a ambiguidade entre controle e liberdade na rede: "These are complex questions which have arisen from this event, one that has been made possible by the dual nature of the Internet that Protocol point to: its horizontality (community networks; TCP/IP) and its verticality (its stratification; DNS)"2 (GALLOWAY, 2004, p. 8). Isso quer dizer que os protocolos TCP/IP, conjunto de regras fundamentais para ligar uma máquina ou uma rede à Internet, permitem a comunicação completamente distribuída. Ao mesmo tempo, o sistema de nomes de domínio (DNS) trabalha de modo extremamente hierárquico. Existe um organismo que define as regras dos domínios de alto nível, uma organização não-governamental, sediada nos Estados Unidos, que recebe influência direta do Departamento de Comércio norteamericano, denominada Icann (Corporação da Internet para a Atribuição de Nomes e Números) (ICANN, 2009). O sistema DNS foi construído com a definição de zonas de conexão ligadas a 13 servidores-raiz (Root Servers). Estes possuem a relação dos nomes de domínios e seus respectivos números IPs em sua área de cobertura.

A observação da arquitetura da Internet, seus protocolos e sua topologia, tem grande importância para a compreensão dos conflitos políticos pelo seu controle ou liberação. Alexander Galloway (2004) não trabalha com o conceito de arquitetura, mas com o de protocolo. Sua visão é inspirada pela perspectiva foucaultiana-deleuziana que articula o período histórico das sociedades de con-

\footnotetext{
2 Tradução livre: "Existem questões complexas que surgiram a partir deste evento, tornado possível graças à dupla natureza dos protocolos da Internet que apontam para: a sua horizontalidade (redes comunitárias; TCP / IP) e sua verticalidade (sua estratificação; DNS)". Podemos definir o acrônimo TCP/IP (Transmission Control Protocol/Internet Protocol) como a reunião de protocolos que definem como a comunicação ocorre na Internet. Alguns técnicos denominam-no "alma da Internet". Já o DNS (Domain Name System) é um sistema para traduzir os endereços de IP em nomes de domínio na rede.
} 
trole com o estilo de gerenciamento protocolar, definido como típico de um diagrama de poder baseado na organização distribuída. Galloway (2004, p. 244) afirmou que é possível prever, no futuro próximo, o confronto entre as duas tendências contrapostas: a do poder centralizado e hierárquico na rede, por um lado, e a sua conexão distribuída, por outro.

Enquanto a ambigüidade da rede vai sendo explorada por forças sociais portadoras de interesses muitas vezes antagônicos, inúmeras batalhas vão ocorrendo simultaneamente em dois planos. Um é o tecno-social, em que o embate dá-se entre a disseminação de tecnologias de controle ou de liberação. Outro é o estritamente jurídicolegislativo, no terreno dos Estados nacionais, apesar das óbvias dificuldades de se controlar dentro das fronteiras de um país as decisões sobre fluxos de informações que são transnacionais. Os conflitos e as políticas da Internet sobre a infraestrutura da rede, seus conteúdos e seus formatos dão-se nos dois planos. A seguir, serão apresentados os traços principais de cada uma dessas três políticas da rede mundial de computadores.

\section{DISPUTAS SOBRE A INFRAESTRUTURA FÍSICA E LÓGICA}

A Internet funciona articulando uma série de camadas lógicas de comunicação sobre uma camada física de infra-estrutura. Cada uma dessas camadas possui inúmeros protocolos que definem como o fluxo de comunicação entre máquinas e redes deve ser estabelecido. Nenhuma camada interfere nos protocolos nem no funcionamento da outra, apenas se comunica com ela. Esse é um princípio que permitiu à rede expandir-se de modo veloz, incorporando novas criações e novas possibilidades de comunicação. Por exemplo, Tim Berners-Lee e outros técnicos criaram o protocolo HTTP (Hypertext Transfer Protocol) que tornou possível a existência da $W e b$, o modo gráfico da rede. Esse protocolo, da chamada "camada de aplicação", apenas deveria comunicar-se com os demais protocolos das outras camadas. Tim Berners-Lee não teve que solicitar às companhias de telecomunicação, que controlam a camada de conexão física da rede, autorização para criar a Web. Uma camada tem autonomia em relação à outra. Este é o arranjo que dirigiu a evolução da Internet, também conhecido como princípio da neutralidade da rede (net neutralit).

Tim Wu, professor da Columbia Law School, argumenta que desde o início dos anos 2000, as operadoras de telecomunicação vêm combatendo o princípio da neutralidade e tratando de modo discriminatório os pacotes de informação que trafegam por suas redes físicas (WU, 2003). Wu denomina discriminação (Broadband Discrimination) a política aplicada pelas operadoras de banda larga. Pelo princípio da neutralidade das camadas da rede, nenhum pacote de informação deve ser tratado de modo diferenciado pelos controladores da rede física, independentemente do endereço IP de origem ou de destino e da aplicação a que pertence. Isso quer dizer que não importa se o pacote contém partes de uma página da Web ou de um correio eletrônico ou de um arquivo de voz sobre IP, entre outras possibilidades.

A disputa aqui é tecnológica e jurídicolegislativa. A tecnológica é travada a partir do desenvolvimento de soluções de controle da rede. As operadoras utilizando em seus roteadores softwares de identificação e rastreamento de pacotes de informação (traffic shapers) conseguem saber que um determinado fluxo entre o IP de origem e o IP de destino trata-se, por exemplo, de uma comunicação de telefonia na internet. Assim, ela simplesmente atrasa a velocidade desses pacotes em sua rede, inviabilizando a comunicação de voz sobre IP. Também podem impedir ou dificultar o uso de redes P2P (STONE, 2007) ao identificar que alguém está compartilhando vídeos ou músicas utilizando sua infra-estrutura (KANG, 2008).

Do ponto de vista jurídico-político, as disputas passam pelos órgãos e agências reguladoras das telecomunicações e também pelas batalhas pela aprovação de leis que garantam ou alterem o princípio da neutralidade na rede ${ }^{3}$. As operadoras de telefonia, nos Estados Unidos, aliadas às indústrias do copyright, têm defendido o fim do princípio da neutralidade da rede e a implantação das regras de livre mercado no ciberespaço. Isso implicaria a alteração do modo como os pacotes são trocados, hoje, na Internet. Os controladores da infraestrutura de conexão poderiam cobrar diferenciadamente pelos pacotes que transitam em

3 Veja o Projeto de Lei do representante democrata de Massachusetts, denominado Internet Freedom Law (INTERNET FREEDOM LAW, 2008). 
suas redes. Atualmente, os pacotes de bits não são precificados de modo diferenciado. A legislação de telecomunicações da maioria dos países garante que todos os pacotes de informação, denominados "datagramas", devem ser tratados do mesmo modo, seja de um sítio eletrônico poderoso ou de um modesto blog, seja de uma grande corporação ou de uma organização não-governamental, seja de uma página $W e b$ ou de uma televisão sobre IP.

O poderoso lobby das operadoras norte-americanas junto aos congressistas para alterar a legislação com a finalidade de implantar as regras de mercado no ciberespaço e, desse modo, destruir o princípio da neutralidade, gerou uma grande reação da sociedade civil naquele país. Uma série de acadêmicos, como Lawrence Lessig, entidades de grande reputação, como a Electronic Frontier Foundation (EFF), personalidades tais como os integrantes da banda pop Pearl Jam e instituições como a FreePress, reuniram-se para formar a coalizão denominada Save the Internet (SAVE THE INTERNET, 2009). Com uma petição que ultrapassou um milhão de assinaturas, o movimento tem o apoio do Presidente eleito Barack Obama, que já era um dos seus integrantes.

A tendência é que a disputa pela alteração da arquitetura da rede intensifique-se quanto mais importante a rede torne-se para as corporações e para o capitalismo. As redes físicas de conexão de alta velocidade, os backbones da Internet, estão nas mãos das operadoras de telecomunicação. Tal fato coloca uma grande preocupação sobre o futuro da comunicação distribuída, pois o controle da infra-estrutura de conexão está concentrado em poucas corporações gigantescas que possuem mais força política que a maioria dos Estados nacionais do planeta. Assim, um dos pontos cruciais das disputas e da política da Internet passa pelas batalhas em torno de sua infra-estrutura de conexão. Outras batalhas ocorrem e ainda ocorrerão no terreno tecnológico em torno da atualização dos protocolos de comunicação, seja das camadas lógicas, seja da camada física da rede.

\section{CONTENCIOSOS SOBRE OS FORMATOS EAPLICAÇÕES}

Um dos principais instrumentos de poder econômico e simbólico na rede está no controle dos formatos e das aplicações. Simultaneamente, uma das principais características da Internet é a grande liberdade para a criação, não somente de conteúdos, mas de novas aplicações e formatos. No mundo digital, os formatos definem como determinado aplicativo irá reconhecer os dados por ele gerados. Aplicação ou aplicativo na rede pode ser compreendido como um programa que desempenha alguma tarefa ou oferece alguma funcionalidade para os internautas. Os aplicativos possuem formatos específicos que podem ser abertos ou fechados, livres ou proprietários, desenvolvidos compartilhadamente ou controlados por uma corporação ou um grupo de empresas.

A Internet possui um modelo de transmissão de dados baseado em uma série de protocolos que podem ser agrupados em camadas de uma pilha. $\mathrm{O}$ termo pilha refere-se a um tipo de estrutura de dados que segue a lógica LIFO (last in, first out), ou seja, uma estrutura de dados que utiliza o procedimento do "último a entrar, primeiro a sair". Os protocolos de comunicação da Internet estão agrupados nas seguintes camadas: camada de aplicação, camada de transporte, camada de rede, camada de enlace e camada física. As camadas mais próximas ao interagente ou usuário da Internet são as mais externas. Assim, a camada de aplicação está mais próxima do internauta enquanto a camada física é a mais distante (NAUGLE, 2001).

A complexidade e a sofisticação da Internet estão nas pontas da rede, na sua periferia, nas camadas mais próximas ao usuário. A pilha TCP/ IP, considerada a alma da Internet, é de simples realização e traz o conceito de redes. Desse modo, para se conectar à Internet era necessário aplicar o TCP/IP em uma determinada rede que permaneceria com sua lógica interna. A simplicidade e acessibilidade do modelo da Internet pode ter sido a principal razão de seu sucesso diante de redes concorrentes, tais como a AOL e o Minitel. Como o modelo de camadas não limita novas aplicações, qualquer interagente pode criar novos protocolos e aplicações, desde que se comunique corretamente com a pilha TCP/IP. Nesse sentido, é correto afirmar que a grande criatividade da rede ocorre nas suas beiradas, na sua periferia. Foi explorando essas possibilidades que Tim Berners-Lee desenvolveu o protocolo HTTP, viabilizando a $\mathrm{Web}$; que Ian Clarke iniciou o projeto Freenet; ou Shawn Fanning criou o Napster, sendo estes dois últimos fundamentais para a explosão das redes $\mathrm{P} 2 \mathrm{P}$. 
Quando falamos de formatos e aplicações necessariamente teremos que recorrer a protocolos e padrões. Na comunicação digital os padrões são essenciais para que os códigos sejam formulados compreensivamente e para que a comunicação ocorra. A estrutura da Internet permite a criatividade e a expansão de aplicações, mas também permite que grandes corporações busquem criar padrões de fato, ou seja, aplicações que dominam o mercado e acabam ditando sua evolução a partir do uso de técnicas de aprisionamento de seus usuários. Isso pode ocorrer pela geração de dependência dos formatos de conteúdos que só podem ser visualizados em um produto ou pela simples adesão de usuários à confortável utilidade de uma determinada aplicação.

Entre as várias batalhas em torno de padrões, formatos e aplicações temos dois exemplos bem ilustrativos da política da rede. O primeiro trata de formatos e ocorreu principalmente no terreno sócio-técnico. O segundo trata de uma aplicação que atualmente lidera o tráfego da Internet, o P2P e diz respeito às disputas jurídico-legislativas.

Um conjunto de empresas, entre elas a $S U N$ Microsystem e a IBM, além de uma série de coletivos de desenvolvimento de software, tais como a comunidade Linux, começaram a desenvolver um formato para documentos, aberto e livre do pagamento de royalties, o OpenDocument Format (ODF). O objetivo era garantir a interoperabilidade e a comunicação plena na armazenagem e troca de documentos, fossem eles textos, planilhas de cálculo, base de dados, gráficos e apresentações. Isso garantiria que um produto abriria um texto escrito em outro produto, ou seja, a interoperabilidade estaria garantida. $\mathrm{O}$ maior beneficiário seria o usuário, pois haveria concorrência de produtos que trabalhariam com um mesmo formato (ODF ALLIANCE, 2009).

O ODF, baseado na linguagem XML, foi aprovado como norma internacional pela International Organization for Standardization (ISO), em maio de 2006 (ISO/IEC 26 300). A ISO, Organização Internacional para Padronização, é uma entidade sediada em Genebra, que congrega os institutos de padronização ou normalização de 160 países (INTERNATIONAL ORGANIZATION FOR STANDARDIZATION, 2009). Por exemplo, o American National Standards Institute (ANSI) é o representante dos Estados Unidos na ISO. A Associação Brasileira de Normas Técnicas
(ABNT) é a representante brasileira. Também em maio de 2006, foi formada a ODF Alliance, consórcio aberto, com a finalidade de manter, aprimorar e disseminar o novo formato.

Temerosa do impacto da interoperabilidade em seus negócios, a Microsoft, junto com o ECMA, consórcio que reúne algumas indústrias européias, preferiram criar um outro formato aberto para documentos (ECMA INTERNATIONAL, 2009). Assim surgiu o Office Open XML Format, também conhecido como Open XML. Contendo mais de 5000 páginas, o formato dificilmente pode ser aplicado corretamente pelos concorrentes dos produtos da Microsoft. O objetivo estratégico da empresa é evitar que o formato $O D F$ disseminasse-se, acirrando a concorrência com um dos seus produtos mais rentáveis, o software Office, suíte que contém o Word, o Excel e o Power Point.

Com dois formatos, tenta-se anular o efeito da concorrência sob um único padrão. As objeções técnicas levantadas contra o Open XML partiam de suas imperfeições e incorreções indo até a crítica mais ampla de que um formato aberto não poderia conter rotinas patenteadas. É interessante notar que apesar da linguagem XML ser aberta desde sua criação, a Microsoft usa a estratégia eufemista de chamar seu formato de Open $X M L$, dando a entender que eles seriam paladinos dos padrões abertos. Todavia, é notório que a Microsoft é uma das principais corporações que se beneficiam do modelo proprietário de códigofonte fechado dos seus programas de computador ${ }^{4}$.

Em uma sociedade em que as redes digitais se transformam nos principais meios de comunicação, o software torna-se também uma mídia, mas uma mídia especial. Quando escrevemos um texto no computador temos que utilizar um software, para enviarmos um e-mail, visualizarmos uma foto digital, ouvirmos uma música ou falarmos no Skype, também utilizamos softwares. Assim, os programas de processamento, os softwares, são

\footnotetext{
4 No sítio eletrônico da Microsoft existem diversas páginas sobre a concepção que embasa o modelo proprietário da empresa: "O software é considerado propriedade intelectual. A propriedade de tal bem é controlada por acordos de licenciamento. As licenças de software são documentos legais que descrevem a correta utilização e distribuição deste software, de acordo com as normas do fabricante" (MICROSOFT, 2009).
} 
intermediários essenciais da comunicação social na era da informação. Ao contrário de suportes como o papel e de instrumento como a caneta, o software pode interferir no conteúdo, na forma de transferi-los e até na capacidade de manter um arquivo no decorrer do tempo. Imagine um texto escrito hoje em um software da Microsoft que irá armazená-lo (salvá-lo) em um formato ".doc". Daqui a dez anos, ao contrário de um texto escrito no papel, qualquer um que buscar lê-lo será obrigado a usar um software compatível com aquele formato. Se a empresa, que é proprietária do formato e do software, tiver alterado seu padrão ou simplesmente não quiser mais mantê-los, dificilmente será possível ler aquele texto no futuro.

A importância dos padrões abertos está na independência diante de corporações, interesses políticos, comerciais ou mesmo ideológicos. Do ponto de vista econômico, a abertura significa a ausência de barreiras para seu uso e aprimoramento, superando as patentes de algoritmo que visam impedir o uso de rotinas lógicas por um dado período; representa, conseqüentemente, uma maior concorrência entre quem deles se utiliza comercialmente. Do ponto de vista científico e tecnológico, garante a democratização do conhecimento sobre seu uso e assegura a realização de experimentos e práticas recombinantes, o que estimula a criatividade e a inventividade. Exatamente devido à inegável vantagem dos padrões abertos é que mesmo as corporações que vivem da negação de acesso ao conhecimento, das rotinas contidas em seus softwares buscam afirmar que defendem a interoperabilidade e a comunicabilidade.

A força de corporações como a Microsoft é tão grande diante da maioria dos Estados nacionais que pode explicar como ela conseguiu em menos de seis meses mudar o voto de diversas organizações nacionais de padronização que haviam se posicionado contra o Open $X M L$, em uma primeira consulta realizada no final de 2007 (ZMOGINSKI, 2008). O Brasil, representado pela ABNT, votou contra o padrão proposto pela Microsoft nas duas consultas, sendo que na primeira delas ofereceu 63 restrições técnicas à sua aprovação (ZMOGINSKI, 2007). Mesmo sem resolver de modo definitivo nenhuma dos graves problemas apontados pelo estudo realizado pelo grupo de trabalho formado pela ABNT, a Microsoft conseguiu os votos necessários para a aprovação da ISO. O governo de George W. Bush, acionado pelo ex-monopólio de software, agiu para mudar o voto da delegação norte-americana e inglesa. Mas nenhum dos representantes de países que formam o chamado BRIC votou a favor do Open XML: Brasil, Índia e China votaram contra, enquanto a Rússia absteve-se.

Para continuar discutindo a política da Internet em torno de padrões, formatos e aplicações será apresentado a seguir um exemplo de outra ordem que se relaciona com uma série de batalhas no plano legislativo e jurídico. Um dos mais importantes conflitos na Internet atualmente diz respeito aos limites do compartilhamento de arquivos digitais. Seu foco está nas redes P2P, que ultrapassam, há algum tempo, mais de $50 \%$ do tráfego da rede mundial de computadores (BANGEMAN, 2007). As aplicações P2P permitem a troca veloz de arquivos digitais e têm sido utilizadas por milhões de internautas para compartilhar músicas, vídeos, imagens e textos. A indústria de copyright, capitaneadas pela RIAA (Recording Industry Association of America) e pela MPAA (Motion Picture Association of America) acusam o P2P de ser o principal meio de disseminação da "pirataria", ou seja, da cópia ilegal de músicas e vídeos.

As redes $\mathrm{P} 2 \mathrm{P}$ possuem arquiteturas diferenciadas, mas, em geral, permitem a troca descentralizada de arquivos pela Internet. Existem arquiteturas P2P que mantém uma estrutura mais centralizada, como o Napster, descentralizadas, tal como o KaZaA, ou completamente distribuídas, como o Gnutella e o BitTorrent. No caso da rede BitTorrent, seu protocolo assegura que os pacotes de um arquivo "baixado" (download) sejam imediatamente oferecidos para upload. Assim, cada computador que entra em uma rede baixando um arquivo musical torna-se imediatamente um servidor dessa mesma música para outros computadores que a buscarem na rede. Essa arquitetura, que torna todo computador, ao mesmo tempo, cliente e servidor de informações permite o compartilhamento veloz e distribuído de arquivos pela rede mundial de computadores.

Um dos principais trackers de BitTorrent é o Pirate Bay (2009). Em uma rede BitTorrent, o tracker é a "denominação dada ao servidor que é responsável por organizar os arquivos disponíveis e direcionar os downloads" (ALECRIM, 2004). 
Em 31 de maio de 2006, a polícia sueca invadiu a empresa que hospedava os servidores do Pirate Bay para apreendê-los. Indexar arquivos não é crime pelas leis suecas, mas a pressão do governo norte-americano levou o governo da Suécia a agir de modo autoritário. No dia 3 de junho de 2006, mais de 600 pessoas realizaram um protesto em Estocolmo contra a ação policial. As manifestações foram organizadas pelo Piratbyrån e pelo Partido Pirata da Suécia, organizações contrárias ao copyright. Um documentário, feito pelos apoiadores do Pirate Bay, chamado Steal this Film ilustra os acontecimentos aqui brevemente relatados (STEAL THIS FILM, 2009).

No dia 17 de abril de 2009, a Justiça da Suécia condenou os fundadores do The Pirate Bay, Gottfrid Svartholm Warg (também conhecido como "Anakata"), Peter Sunde Kolmisoppi ("Brokep") e Fredrik Neij ("TiAMO"), por facilitar a violação de copyright. Os jovens foram processados pelas corporações da indústria de intermediação cultural: Warner Bros, MGM, EMI, Columbia Pictures, 20th Century Fox, Sony BMG e Universal (VALENTIN, 2009). O julgamento foi acompanhado por milhões de internautas e teve um desfeito juridicamente controverso. Dias depois, descobriu-se que o juiz que condenou os jovens do The Pirate Bay integrava a mesma associação de defesa do copyright que o promotor. Apesar da condenação, o processo aparentemente serviu para disseminar uma idéia positiva do P2P para uma parcela expressiva da opinião pública transnacional. Baseado no resultado político e social do processo movido contra o Napster, em 2001, nada indica que o compartilhamento de arquivos realizado pelos 22 milhões de usuários do Pirate Bay poderá ser bloqueado por medidas judiciais.

Percebendo tal possibilidade, a RIAA, que desenvolve uma extensa campanha doutrinária contra as redes P2P combinada com processos judiciais contra milhares de internautas, quer mudar a estratégia de combate. Seu objetivo é buscar acordos com provedores de acesso à Internet com a finalidade de bloquear o uso do P2P entre seus usuários (ANDERSON, 2008). Ao mesmo tempo, a indústria de copyright conseguiu na França um grande trunfo contra as aplicações P2P. O presidente Nicolas Sarkozy aprovou uma lei que permite aos provedores de acesso franceses desconectarem, por um período de tempo que varia de três meses a um ano, quem utilizar as redes $\mathrm{P} 2 \mathrm{P}$ para compartilhar músicas cerceadas pelo copyright (EL PAÍS, 2008). O grande problema é que a única forma dos provedores saberem se os arquivos compartilhados pelo protocolo P2P são ou não ilegais é violando o sigilo da comunicação e observando o conteúdo que está sendo transferido. Para Sarkozy e para a indústria de copyright, a privacidade dos cidadãos parece ser completamente secundária.

\section{CONCLUSÕES: TENTATIVAS DE CONTRO- \\ LE DOS CONTEÚDOS E AS ROTAS DE FUGA}

Existe uma pressão de parte da direita mundial e de alguns segmentos da antiga esquerda, principalmente depois dos atentados de 11 de setembro, pelo fim da comunicação anônima na Internet. Essa pressão é ampliada pelos políticos conservadores que superdimensionam crimes hediondos, tais como a pedofilia, para conseguir apoio público visando restringir as liberdades na rede. Todavia, os protocolos de comunicação da rede asseguraram o anonimato. Alexander Galloway afirmou que os protocolos não são simples regras: na comunicação cibernética, são como "pegadas deixadas na neve" (GALLOWAY, 2004, p. 244). Nas redes informacionais, a comunicação deixa um rastro digital. Esse rastro tem sido utilizado pelas empresas de vigilância, de pesquisa, de marketing, de seguros, de relações públicas e, também, pelos Estados autoritários para pesquisar, controlar e até perseguir os internautas.

O governo da China, uma ditadura, exige que todos os usuários da Internet tenham um registro na polícia. Ao contrário do que ocorre até o momento no Brasil, onde é possível acessar redes abertas sem a necessidade de identificação, em vários países autoritários o anonimato não é possível. A comunicação anônima é considerada criminosa, o que significa que todo endereço IP deve estar vinculado a uma identidade pessoal. No fundo, o que o pensamento autoritário quer impedir é a comunicação distribuída consolidada pela Internet, pois ela impede o controle dos conteúdos e dificulta a imediata identificação e a ação sobre o interagente. Esse pensamento é contrário ao estabelecimento de uma esfera pública interconectada baseada na reputação de avatares e codinomes, construída sobre a força dos argumentos, independentemente da identidade do falante.

Yochai Benkler escreveu que a esfera pública interconectada é mais democrática do que a esfera pública controlada pelos meios de comunica- 
ção de massa, primeiramente por reduzir os custos de se tornar um falante, e depois por distribuir completamente a possibilidade de participar dos debates devido à arquitetura da rede (BENKLER, 2006). Na Internet, ninguém precisa de autorização para criar conteúdos, para tal basta criar um sítio eletrônico, um portal ou um blog. Todavia, como a tecnologia da informação é ambígua, a comunicação distribuída também pode ser utilizada para difamar e para beneficiar articulações criminosas. A solução proposta pelo pensamento autoritário, originado na direita ou na esquerda, passa pela alteração do funcionamento atual da Internet, ampliando as regras de segurança e diminuindo a liberdade dos fluxos, aumentando as possibilidades de controle da navegação e reduzindo o terreno da privacidade dos interagentes.

Os arquitetos da Internet, influenciados pela cultura hacker (CASTELLS, 2003), desenvolveram protocolos da rede que asseguram a liberdade da comunicação. A idéia era proteger a livre expressão de quaisquer tipos de pressão política, religiosa, ideológica, profissional, corporativa, pública ou privada. Muito influenciada pela contracultura norte-americana, a arquitetura da Internet foi desenhada para preservar a comunicação sem a necessária identificação do sujeito comunicante, pois isso é considerado a condição do direito à opinião. Nesse sentido, Ian Clarke, criador e programador principal da rede Freenet, um típico hacker, no texto The Philosophy Behind Freenet, esclarece: "But why is anonymity necessary?

You cannot have freedom of speech without the option to remain anonymous. Most censorship is retrospective, it is generally much easier to curtail free speech by punishing those who exercise it afterward, rather than preventing them from doing it in the first place. The only way to prevent this is to remain anonymous. It is a common misconception that you cannot trust anonymous information. This is not necessarily true, using digital signatures people can create a secure anonymous pseudonym which, in time, people can learn to trust. Freenet incorporates a mechanism called "subspaces" to facilitate this" (CLARKE, 2009).

$\mathrm{O}$ argumento hacker sobre a verdade e a verificação dos conteúdos anônimos passa pelos mecanismos de reputação, de denúncia colaborativa e pelas redes de confiança. Mais do que isso, a garantia da liberdade, nas redes cibernéticas, de produzir conteúdos e de acessá-los - com os rastros digitais acompanhando toda a navegação dos cidadãos interagentes - exige a garantia do anonimato como seu elemento basilar e como uma contraposição ao poder de controle. Esse é um dos principais contenciosos da política da Internet.

Sergio Amadeu da Silveira (sergioamadeu@uol.com.br)é Doutor em Ciência Política pela Universidade de São Paulo (USP) e Professor do Mestrado em Comunicação da Faculdade Cásper Líbero.

\section{REFERÊNCIAS BIBLIOGRÁFICAS}

ALECRIM, E. 2004. BitTorrent - o que é e como funciona. Infowester, 19.set. Disponível em : http://www.infowester.com/bittorrent.php. Acesso em : 20.fev.2009.

ANDERSON, N. 2008. No More Lawsuits : ISPs to Work With RIAA, Cut Off P2P Users. Ars Technica, Chicago, 19.Dec. Disponível em : http://arstechnica.com/tech-policy/news/2008/ 12/no-more-lawsuits-isps-to-work-with-riaacut-off-p2p-users.ars. Acesso em : 22.fev. 2009 .

BANGEMAN, E. 2007. P2P Responsible for as much as 90 Percent of All 'Net Traffic'. Ars Technica, Chicago, 2.Sept. Disponível em : http://arstechnica.com/old/content/2007/09/ p2p-responsible-for-as-much-as-90-percentof-all-net-traffic.ars. Acesso em : 22.fev.2009.

BARBER, B. 2004. Which Technology and Which Democracy? In : JENKINS, H. \& THORBURN, D. (org.). Democracy and New Media. Cambridge, MA. : MIT.

BARROS FILHO, C.; COUTINHO, M. \& SAFATLE, V. 2007. Os usos das novas mídias na campanha presidencial de 2006. In : LIMA, V. A mídia nas eleições de 2006. São Paulo : P. Abramo.

BENKLER, Y. 2006. The Wealth of Networks : How Social Production Transforms Markets And Freedom. New Haven : Yale University. 
CASTElLS, M. 1999. A sociedade em rede. A era da informação : economia, sociedade e cultura. V. I. São Paulo : Paz e Terra.

2003. A galáxia da Internet : reflexões sobre a Internet, os negócios e a sociedade. Rio de Janeiro : J. Zahar.

CHAIA, V. 2008. Internet e eleições na Espanha. Observatório da imprensa, São Paulo, 12.fev. Disponível em: http://www.observatorioda imprensa.com.br/artigos.asp? cod= 472ENO002. Acesso em : 2.ago.2009.

CLARKE, I. The Philosophy Behind Freenet. Disponível em : http://freenetproject.org/ philosophy.html. Acesso em : 10.fev.2009.

COLEMAN, S. 1999. Cutting out the Middle Man : from Virtual Representation to Direct Deliberation. In : HAGUE, B. \& LOADER, B. (org.). Digital Democracy : Discourse and Decision Making in the Information Age. New York : Routledge.

GALLOWAY, A. 2004. Protocol: How Control Exists After Decentralization. Cambridge, MA. : MIT.

HABERMAS, J. 1984. Mudança estrutural da esfera pública : investigações quanto a uma categoria de sociedade burguesa. Rio de Janeiro : Tempo Brasileiro.

HAGUE, B. \& LOADER, B. 1999. Digital Democracy : an introduction. In : HAGUE, B. \& LOADER, B. (org.). Digital Democracy : Discourse and Decision Making in the Information Age. New York : Routledge.

JENKINS, H. \& THORBURN, D. 2004. Introdution : the Digital Revolution, the Informed Citizen, and the Culture of Democracy. In : JENKINS, H. \& THORBURN, D. (org.). Democracy and New Media. Cambridge, MA. : MIT.

KANG, C. 2008. Comcast Defends Role As Internet Traffic Cop. The Washington Post, 13.Feb, p. D01.

KOHUT, A. 2008. The Internet Gains in Politics. Pew Internet, Washington, D.C., 1.Nov. Disponível em : http://www.pewinternet.org/PPF/ r/234/report_display.asp. Acesso em : 20.fev.2009.
LESSIG, L. 1999. Code and Other Laws of Cyberspace. New York : B. Books.

NAUGLE, M. 2001. Guia Ilustrado do TCP/IP. São Paulo : Berkeley Brasil.

NIXON, P. \& JOHANSSON, H. 1999. Transparency Through Technology : the Internet and the Political Parties. In : HAGUE, B. \& LOADER, B. (org.). Digital Democracy: Discourse and Decision Making in the Information Age. New York : Routledge.

NOVECK, B. 2005. A Democracy of Groups. First Monday, v. 10, n. 11, 7.Nov. Disponível em : http://firstmonday.org/htbin/cgiwrap/bin/ ojs/index.php/fm/article/view/1289/1209. Acesso em : 18.Jan.2009.

PISCITELLI, A. 2002. Ciberculturas 2.0 : en la era de las máquinas inteligentes. Buenos Aires : Paidós.

RHEINGOLD, H. 2004. Multitudes inteligentes: la próxima revolución social. Barcelona : Gedisa.

RIBEIRO, G. 2000. Política cibercultural : ativismo político à distância na comunidade transnacional imaginada-virtual. In :ALVAREZ, S.; DAGNINO, E. \& ESCOBAR, A. Cultura e política nos movimentos sociais latino-americanos. Belo Horizonte : UFMG.

SILVEIRA, S. 2007. Combates na fronteira eletrônica : a Internet nas eleições de 2006. In : LIMA, V. A mídia nas eleições de 2006. São Paulo : P. Abramo.

STONE, B. 2007. Comcast : We're Delaying, Not Blocking, BitTorrent Traffic. The New York Times, Bits, 22.Oct.2007. Disponível em : http:// bits.blogs.nytimes.com/2007/10/22/comcastwere-delaying-not-blocking-bittorrent-traffic/. Acesso em : 20.fev.2009.

TERRANOVA, T. 2004. Network Culture : Politics for the Information Age. London : Pluto.

UGARTE, D. 2008. O poder das redes. Porto Alegre : PUC-RS.

VALENTIN, D. 2009. The Pirate Bay Trial. Blog Lalai Loaded, 18.fev. Disponível em : http:// lalai.net/2009/02/18/the-pirate-bay-trial/. Acesso em : 28.fev.2009. 
WILHELM, A. 1999. Virtual Sounding Boards : How Deliberative is Online Political Discussion? In : HAGUE, B. \& LOADER, B. (org.). Digital Democracy: Discourse and Decision Making in the Information Age. New York : Routledge.

WU, T. 2003. Network Neutrality, Broadband Discrimination. Journal of Telecommunications and High Technology Law, Boulder, v. 2, p. 141.
ZMOGINSKI, F. 2007. Brasil dirá não ao Open XML na ISO, diz ABNT. Plantão Info, São Paulo, 23.ago. Disponível em : http:// info.abril.com.br/aberto/infonews/082007/ 23082007-14.shl. Acesso em : 22.fev.2009.

2008. ISO oficializa aprovação do Open XML. Plantão Info, São Paulo, 2.abr.2008. Disponível em : http://info.abril.com.br/aberto/infonews/042008/02042008-0.shl. Acesso em : 20.fev.2009.

\section{OUTRAS FONTES}

ECMA INTERNATIONAL. 2009. TC45 - Office Open XML Formats. Disponível em : http:// www.ecma-international.org/memento/ TC45.htm. Acesso em : 10.fev.2009.

Francia inicia el camino para desconectar de Internet a quien realice descargas ilegales. 2008. El País.com, Madrid, 18.jun. Disponível em : http://www.elpais.com/articulo/internet/ Francia/inicia/camino/desconectar/Internet/ quien/realice/descargas/ilegales/elpeputec/ 20080618elpepunet_8/Tes. Acesso em : 25.fev.2009.

ICANN. 2009. Sítio eletrônico. Disponível em : http://www.icann.org/. Acesso em : 02.ago.2009.

INTERNATIONAL ORGANIZATION FOR STANDARDIZATION. 2009. About ISO. Disponível em : http://www.iso.org/iso/about. Acesso em : 10.fev.2009.

Internet Freedom Law Will Keep Internet Open for Future Innovators. 2008. Congressman Edward Markey, Washington, D.C., 12.Feb.
Disponível em : http://markey.house.gov/ index.php?option $=$ com_content\&task $=$ view\&id=3268\&Itemid=141. Acesso em : 25.fev.2009.

MICROSOFT. 2009. A importância do licenciamento. Disponível em : http:// www.microsoft.com/brasil/antipirataria/ how licenses.mspx. Acesso em : 24.mai.2009.

ODF ALLIANCE. 2009. Sítio eletrônico .Disponível em : http://www.odfalliance.org/. Acesso em : 10.fev.2009.

PIRATE BAY. 2009. Sítio eletrônico. Disponível em : http://trial.thepiratebay.org/. Acesso em : 2.mar.2009.

SAVE THE INTERNET. 2009. Sítio eletrônico. Disponível em : http://www.savethe internet.com/. Acesso em : 12.fev.2009.

STEAL THIS FILM. 2009. Sítio eletrônico. Disponível em : http://www.stealthisfilm.com/ Part2/download.php. Acesso em : 28.fev.2009. 


\section{NEW DIMENSIONS OF POLITICS: PROTOCOL AND CODES IN THE "CONNECTED" PUBLIC SPHERE}

\section{Sérgio Amadeu da Silveira}

This text proposes the existence of a basic divide in the relationship between politics and the Internet: the politics "of" the Internet and politics "on" the Internet. Topics of the politics of the internet are then divided into three fundamental fields of contention: network infrastructure; formats, patterns and applications and contents. It analyzes the most relevant current political topics within each field of conflict, articulating two aspects: the techno-social and the juridical-legislative. This article works with the concept of the architecture of power, an extension of Alexander Galloway's definition of protocol management and with Yochai Benkler's perspective on the existence of an interconnected public sphere. We conclude that freedom within cybernetic networks depends on the existence of non-identified navigation, that is, of anonymity. Pointed out as one of the major political disputes involving the Internet, the possibility of anonymity is presented as a fundamental element of resistance in the face of the ample possibilities that exist for control of social communication. Thus, to guarantee the freedom to produce content and have access to it through cybernetic networks (in which digital footprints accompany all navigation done by interacting citizens) demands support for non-identification.

Keywords: Internet politics; architecture of power; cyberpolitics; network power. 


\section{DE NOUVELLES DIMENSIONS DE LA POLITIQUE : PROTOCOLES ET CODES DANS L'ESPACE PUBLIC INTERCONNECTÉ}

Sérgio Amadeu da Silveira

Le texte propose l'existence d'une division fondamentale dans la relation entre politique et Internet : la politique « d'Internet » et la politique « sur Internet ». Ensuite, l'article réunit les thèmes des politiques d'Internet en trois terrains de disputes essentielles : l'infrastructure du réseau ; les formats, standards et applications ; et les contenus. Il examine les thèmes politiques actuels les plus marquants de chaque terrain de conflit, en articulant deux aspects : le technosocial et le juridico-législatif. Cet article utilise le concept d'architecture de pouvoir, un prolongement de la définition d'Alexander Galloway sur la gérance protocolaire, et la perspective d'Yochai Benkler sur l'existence d'une dimension publique interconnectée. Sa conclusion indique que la liberté dans les réseaux cybernétiques dépend de l'existence de la navigation non-identifiée, c'est-à-dire de l'anonymat. Considérée comme l'une des principales querelles politiques d'Internet, la possibilité de l'anonymat est présentée comme un élement essentiel de résistance face aux vastes possibilités de contrôle de la commuication sociale. Ainsi, la garantie de la liberté de produire des contenus et de les accéder dans les réseaux cybernétiques sur lesquels les empreintes numérisées suivent toute la navigation des citoyens interagents - exige la défense de la non-identification.

MOTS-CLÉS : politique d'Internet ; architectures du pouvoir ; cyberpolitique ; pouvoir dans les réseaux. 